PROCEEDINGS OF THE

AMERICAN MATHEMATICAL SOCIETY

Volume 135, Number 1, January 2007, Pages 69-75

S 0002-9939(06)08444-9

Article electronically published on July 28, 2006

\title{
ESTIMATES FOR DOMAINS OF LOCAL INVERTIBILITY OF DIFFEOMORPHISMS
}

\author{
OCTAVIAN G. MUSTAFA AND YURI V. ROGOVCHENKO \\ (Communicated by Carmen C. Chicone)
}

\begin{abstract}
Using a novel Wintner-type formulation of the classical Peano's existence theorem [Math. Ann. 37 (1890), 182-228], we enhance Ważewski's result on invertibility of maps defined on closed balls [Ann. Soc. Pol. Math. 20 (1947), 81-125] securing the size of the domain of invertibility that agrees with the bounds derived by John [Comm. Pure Appl. Math. 21 (1968), 77-110] and Sotomayor [Z. Angew. Math. Phys. 41 (1990), 306-310].
\end{abstract}

\section{INTRODUCTION}

In 1906, J. Hadamard [7 established a global version of the inverse function theorem and provided sufficient conditions for a given map to be a diffeomorphism (a differentiable map between manifolds which is one-to-one, onto and whose inverse is differentiable); see also Gordon [6].

Theorem 1. Let $f$ be a map from $\mathbb{R}^{n}$ onto $\mathbb{R}^{n}$ and the derivative $f^{\prime}(x)$ be invertible for every $x \in \mathbb{R}^{n}$. If

$$
\int_{0}^{+\infty} \inf _{\|y\| \leq u} \frac{1}{\left\|f^{\prime}(y)^{-1}\right\|} d u=+\infty
$$

then $f$ is a diffeomorphism of $\mathbb{R}^{n}$ to $\mathbb{R}^{n}$.

Since then, the problem of finding the inverse map, estimating the domain of local invertibility for a smooth function, as well as obtaining conditions for global invertibility of smooth and even nonsmooth maps both in finite-dimensional and infinite-dimensional cases attracted significant interest of the researchers. Elevated interest to global invertibility results reflects their importance for electrical network theory, theory of economic equilibria, stability of nonlinear differential equations, nonlinear elasticity, partial differential equations, etc. A variant of Theorem 1 was discovered by Hadamard [7, extended by Lévy [12, and generalized later by

Received by the editors June 23, 2005.

2000 Mathematics Subject Classification. Primary 34A12, 57R50.

Key words and phrases. Nonlinear differential equations, Peano's existence theorem, regularity, diffeomorphism, local invertibility, Ważewski's theorem, Hadamard-Lévy-Plastock condition.

This research was supported in part by the Mathematisches Forschungsinstitut Oberwolfach, Germany through the Program "Research in Pairs" (O.M. and Y.R.) and by the Abdus Salam International Centre for Theoretical Physics, Trieste, Italy, through the Young Collaborators Program (O.M.) and the Associateship Scheme (Y.R.).

(C)2006 American Mathematical Society Reverts to public domain 28 years from publication 
Plastock 20 to include, in addition to the version of Theorem 1 referring to diffeomorphism between Banach spaces, the Banach-Mazur theorem [1] and Browder's theorem [2]. Furthermore, Plastock noticed that for finite-dimensional spaces, condition (1.1) can be replaced by

$$
\int_{0}^{+\infty} \inf _{\|y\|=u} \frac{1}{\left\|f^{\prime}(y)^{-1}\right\|} d u=+\infty
$$

For other related results we refer the reader to the papers by John [10, Li et al. [13, Porciau [21, Sotomayor [23], Zampieri 27.

A direct approach to the problem of global invertibility requires a delicate analysis of topological and integrability properties. On the other hand, employment of differential equations for establishing invertibility, and vice versa, proved to be very beneficial; see De Marco et al. [14. In fact, Sotomayor [23, Ważewski [25], and Zampieri [27] successfully exploited differential equations for obtaining the estimates for the domain of invertibility of a given map. Conversely, Gasull et al. [5], Olech [17, and Zampieri and Gorni [28] utilized invertibility to prove global stability.

Our research has been stimulated by an ingenious idea of T. Ważewski 25] to examine the properties of solutions of the differential equation

$$
x^{\prime}=\left(f^{\prime}(x)^{-1}\right) v,
$$

where $f$ is a continuously differentiable map from $\mathbb{R}^{n}$ into itself and $v \in \mathbb{R}^{n}$ for obtaining an estimate for the domain of invertibility. Equation (1.2), known nowadays as the Wazewski equation, is well defined in any domain where $f^{\prime}(x)$ is nonsingular, and its solution $x(t)$ is the function whose image is linear,

$$
f(x(t))=f(x(0))+v t .
$$

As emphasized by Olech [18, (1.2) helps to attack the Markus-Yamabe Fundamental Problem on Global Asymptotic Stability of the autonomous system in the plane [15. which was solved by several authors using lengthy and complicated arguments.

In 1990, studying maximal star-shaped domain $S(0)$ of right-invertibility for the mapping $f$ on $\mathbb{R}^{n}$, Sotomayor 23 improved Ważewski's theorem, obtaining results closely related to John's work [10] where maximal ball instead of maximal starshaped domain was used. In particular, employing a modified Ważewski equation,

$$
x^{\prime}=\delta(x)\left(f^{\prime}(x)^{-1}\right) v,
$$

where $\delta$ is a smooth function such that, given a compact set $M \subset \mathbb{R}^{n}$ with $0 \in M$ and $f^{\prime}(x) \neq 0$ in $\bar{M}$, one has $\delta(x)>0$ if $x \in M, \delta(x)=0$ if $x \in \partial M$, and $\delta(x)<0$ otherwise, Sotomayor obtained integral representation for the radial function $\rho(v)$ naturally associated with the star-shaped domain of invertibility. Namely, if $x(t, v)$ is a solution of (1.3) that satisfies the initial condition $x(0, v)=0$, then

$$
\rho(v)=\int_{0}^{+\infty} \delta(x(t, v)) d t, \quad\|v\|=1, v \in \mathbb{R}^{n} .
$$

Stability properties of the radial function $\rho(v)$ with respect to smooth deformations, called $S(0)$-stability of $f$, were studied by Sotomayor [24, Section 2] using the techniques from the singularity theory and a modified Ważewski equation,

$$
x^{\prime}=\left(f^{\prime}(x)^{-1}\right)\left[f(x)-f\left(x_{0}\right)\right] .
$$


Replacing $M$ with a closed ball $D$ of the radius $b>0$ centered at 0 and assuming that

$$
\theta(r)=\sup _{\|x\| \leq r}\left(\limsup _{u \rightarrow 0} \frac{\left\|u-f^{\prime}(x) u\right\|}{\|u\|}\right)<1, \quad 0 \leq r \leq b,
$$

in $D$, one obtains an interesting estimate, discovered by Nevanlinna [16, for the radius of a closed ball on which the function $f$ is invertible, namely

$$
\rho(v)=b-\int_{0}^{b} \theta(r) d r
$$

In this paper, we use the technique developed by Hartman [9, pp. 11-12], in particular, an illuminating hint to the proof of Ważewski's theorem [9] p. 557], and a Wintner-type existence theorem to enhance Ważewski's result [25] magnifying the constants $b M^{-1}$ and $b\left(M M_{1}\right)^{-1}$ up to the values provided by the bounds for the domain of invertibility derived by John [10] and Sotomayor [23].

\section{Peano's existence theorem Revisited}

Peano's existence theorem [19] is one of the key results in the theory of differential equations which, owing to the weakness of assumptions and transparency of the idea underlying its proof, is included in many modern texts on differential equations. An important problem related to Peano's existence theorem considers the possibility for improving the estimate for the interval of existence of a solution; see the fundamental paper by Wintner [26].

In 1988, Lee and O'Regan [11] studied the initial value problem

$$
\left\{\begin{array}{l}
x^{\prime}=f(t, x), \quad t \geq 0, x \in \mathbb{R}^{n}, \\
x(0)=x_{0},
\end{array}\right.
$$

under the assumption that $f:[0,+\infty) \times \mathbb{R}^{n} \rightarrow \mathbb{R}^{n}$ is a continuous function satisfying $\|f(t, x)\| \leq \psi(\|x\|)$, where $\psi: \mathbb{R}_{+} \rightarrow(0,+\infty)$ is a certain continuous function. They have established the existence of a solution $x(t)$ of the problem (2.1) defined on the interval $[0, T]$, where $T$ is given by

$$
T<T_{\infty}=\int_{\left\|x_{0}\right\|}^{+\infty} \frac{d u}{\psi(u)} \leq+\infty
$$

Our approach to the problem slightly differs from that of Lee and O'Regan and relies on the Leray-Schauder alternative [4, Theorem 5.3, pp. 61-62], also known as Schaefer's theorem [3, Theorem 4.4, pp. 133-134]. We recall that the operator $T: E \rightarrow \mathcal{N}$ defined on a subset $E \subset \mathcal{N}$ is completely continuous (compact) if $T$ is continuous and for a bounded subset $M \subset E$ the set $T(M)$ is relatively compact; see [3, p. 131].

Theorem 2. Let $T: \mathcal{N} \rightarrow \mathcal{N}$ be a completely continuous operator acting on a normed linear space $\mathcal{N}$ and $\lambda_{0} \in[0,1]$. Then either there is an $x \in \mathcal{N}$ such that

$$
x=\lambda_{0} T(x)
$$

or, for any $\lambda \in(0,1)$, the set

$$
E(T)=\{x \in X: x=\lambda T(x)\}
$$

is not bounded. 
Let $a, b>0, x_{0} \in \mathbb{R}^{n}$, and $f: \mathbb{R} \times \mathbb{R}^{n} \rightarrow \mathbb{R}^{n}$ be a continuous function. Consider the initial value problem

$$
x^{\prime}=f(t, x), \quad x\left(t_{0}\right)=x_{0}
$$

on the "box" $B$ defined by

$$
B=\left\{\left|t-t_{0}\right| \leq a, \quad\left\|x-x_{0}\right\| \leq b\right\} .
$$

Introduce a majorizing function $\psi:[0, b] \rightarrow[0,+\infty)$ by the formula

$$
\psi(r)=\sup \left\{\|f(t, x)\|:\left|t-t_{0}\right| \leq a,\left\|x-x_{0}\right\| \leq r\right\}, \quad 0 \leq r \leq b .
$$

Note that $\psi(0)=0$ if and only if (2.3) has the constant solution $x(t)=x_{0}$ on the interval $\left[t_{0}-a, t_{0}+a\right]$. Assuming now that $\psi(0)>0$, we introduce the function $G:[0, b] \rightarrow[0,+\infty)$ by the formula

$$
G(v)=\int_{0}^{v} \frac{d u}{\psi(u)}, \quad 0 \leq v \leq b .
$$

Theorem 3 (Peano's existence theorem). Consider the initial value problem (2.3) on the box $B$. Then either

(i) $\psi(0)=0$ and $x(t)=x_{0}$ is a solution of the problem (2.3) defined on the interval $\left[t_{0}-a, t_{0}+a\right]$, or

(ii) $\psi(0)>0$, and there exists a nonconstant solution of the problem (2.3) defined on the interval $\left[t_{0}-\tau, t_{0}+\tau\right]$, where $\tau=\min [a, G(b)]$.

Proof. Without loss of generality, assume that $x_{0}=0$ and $\psi(0)>0$. Let $\mathcal{N}=$ $C\left(\left[t_{0}-\tau, t_{0}+\tau\right], \mathbb{R}^{n}\right)$ be a Banach space of continuous functions endowed with the standard sup-norm and let $T$ be defined by

$$
T(x)(t)=\int_{t_{0}}^{t} f(s, x(s)) d s, \quad x \in \mathcal{N}, t \in\left[t_{0}-\tau, t_{0}+\tau\right] .
$$

Following Hale [8, Theorem 1.1, p. 15], one can deduce that the operator $T$ is completely continuous. We claim that the set $E(T)$ is bounded. Indeed, for $u \in$ $E(T)$, it follows from the equation

$$
u(t)=\lambda \int_{t_{0}}^{t} f(s, u(s)) d s, \quad\left|t-t_{0}\right| \leq \tau
$$

that

$$
\|u(t)\| \leq \int_{t_{0}}^{t} \psi(\|u(s)\|) d s
$$

for all $t \in\left[t_{0}, t_{0}+\tau\right]$ and for any $\lambda \in(0,1)$. Denote the right-hand member of (2.6) by $z(t)$. Obviously,

$$
z^{\prime}(t) \leq \psi(z(t))
$$

Integrating the latter inequality over $\left[t_{0}, t\right]$, we conclude that

$$
\int_{0}^{z(t)} \frac{d u}{\psi(u)} \leq t-t_{0} \leq \tau
$$

for all $t$ in $\left[t_{0}, t_{0}+\tau\right]$. Note that (2.7) also holds for all $t \in\left[t_{0}-\tau, t_{0}\right]$ if one chooses

$$
z(t)=\int_{t}^{t_{0}} \psi(\|u(s)\|) d s
$$


Taking into account that (2.7) can be written as

$$
\|u(t)\| \leq z(t) \leq G^{-1}(\tau) \leq b, \quad\left|t-t_{0}\right| \leq \tau,
$$

the conclusion follows by application of the Leray-Schauder alternative.

\section{WAŻEWSKI'S RESULT AND BOUNDS FOR THE DOMAIN OF INVERTIBILITY}

Noticing that

$$
\inf _{\|y\| \leq u} \frac{1}{\left\|f^{\prime}(y)^{-1}\right\|}=\frac{1}{\sup _{\|y\| \leq u}\left\|f^{\prime}(y)^{-1}\right\|},
$$

we write the Hadamard-Lévy-Plastock condition (1.1) in the equivalent form

$$
\int_{0}^{+\infty} \frac{d u}{\sup _{\|y\| \leq u}\left\|f^{\prime}(y)^{-1}\right\|}=+\infty .
$$

This representation helps to reveal the link between invertibility of smooth functions and a Wintner-type result (Theorem 3) established by using the comparison function $\psi$ defined by (2.4).

Theorem 4. Let $n \geq 1$ and let $G \subseteq \mathbb{R}^{n}$ be an open set containing the closed ball $D$ of radius $b>0$ centered at 0 . Suppose further that $f: G \rightarrow \mathbb{R}^{n}$ is a continuously differentiable function such that $f(0)=0$ and $\operatorname{det}\left[f^{\prime}(y)\right] \neq 0$, for all $y \in D$. Consider the sets

$$
D_{0}=\left\{x \in \mathbb{R}^{n}:\|x\| \leq \Omega(b)\right\} \quad D_{2}=\left\{y \in \mathbb{R}^{n}:\|y\| \leq M_{1}^{-1} \Omega(b)\right\},
$$

where

$$
\Omega(b)=\int_{0}^{b} \inf _{\|y\| \leq u} \frac{1}{\left\|f^{\prime}(y)^{-1}\right\|} d u
$$

and

$$
M_{1}=\max _{y \in D}\left\|f^{\prime}(y)\right\| .
$$

Then there exists a set $D_{1}, D_{2} \subseteq D_{1} \subseteq D$, such that $f: D_{1} \rightarrow D_{0}$ is a diffeomorphism.

Proof. Consider the initial value problem

$$
\left\{\begin{array}{l}
\frac{d y}{d t}=\left(f^{\prime}(y)^{-1}\right) \xi \\
\|y\| \leq b, \quad|t| \leq 1 \\
y(0)=0
\end{array}\right.
$$

where $\xi \in \mathbb{R}^{n} \backslash\{0\}$ is a fixed vector such that $\|\xi\| \leq \Omega(b)$. Introduce the function $\psi:[0, b] \rightarrow[0,+\infty)$ by

$$
\psi(r)=\|\xi\| \sup \left\{\left\|f^{\prime}(y)^{-1}\right\|:\|y\| \leq r\right\} .
$$

It follows from (3.1) that

$$
G(b)=\|\xi\|^{-1} \int_{0}^{b} \frac{d u}{\sup _{\|y\| \leq u}\left\|f^{\prime}(y)^{-1}\right\|} \geq 1,
$$


and Theorem 3 yields existence of a solution $y(t ; \xi)$, not necessarily unique, of the problem (3.3), which is defined on $[-1,1]$. Writing (3.3) in the form

$$
\left\{\begin{array}{l}
f^{\prime}(y(t ; \xi)) \frac{d y(t ; \xi)}{d t}=\xi, \quad|t| \leq 1, \\
y(0)=0,
\end{array}\right.
$$

we conclude, upon integration, that

$$
f(y(t ; \xi))=t \xi, \quad|t| \leq 1 .
$$

Now the set $D_{0}$ can be redefined as

$$
D_{0}=\left\{t \xi:(t, \xi) \in \mathbb{R} \times \mathbb{R}^{n},|t| \leq 1,\|\xi\| \leq \Omega(b)\right\} .
$$

Clearly, $D_{0} \subseteq f(D)$. Application of the inverse function theorem (see, for instance, [4, Theorem 4.1, pp. 28-30] or [22, Theorem 9.24, pp. 221-223]) yields the existence of the unique solution $y(t ; \xi)$ of (3.4). Letting $g(\xi)=y(1 ; \xi)$, we conclude that the function $g: D_{0} \rightarrow D$ is the inverse of $f$ and $g$ is continuously differentiable.

Consider now the initial value problem

$$
\left\{\begin{array}{l}
\frac{d x}{d s}=\left(g^{\prime}(x)^{-1}\right) \eta \\
\|x\| \leq \Omega(b), \quad|s| \leq 1, \\
x(0)=0
\end{array}\right.
$$

where $\eta \in \mathbb{R}^{n} \backslash\{0\}$ is a fixed vector satisfying $\|\eta\| \leq M_{1}^{-1} \Omega(b)$. Since, for all $z \in D_{0}$, one has $f^{\prime}(g(z)) g^{\prime}(z)=I_{n}$, where $I_{n}$ stands for an $n \times n$ identity matrix, we can choose $\psi(r)=\|\eta\| M_{1}$. Taking into account that

$$
G(\Omega(b))=\|\eta\|^{-1} \int_{0}^{\Omega(b)} \frac{d u}{M_{1}} \geq 1,
$$

we deduce from Theorem 3 and the inverse function theorem that there exists a unique solution $x(s ; \eta)$ of the problem (3.5) defined on the interval $[-1,1]$. Finally, it follows from the relation

$$
g(x(s ; \eta))=s \eta, \quad|s| \leq 1,\|\eta\| \leq M_{1}^{-1} \Omega(b),
$$

which is equivalent to

$$
f(s \eta)=x(s ; \eta)
$$

that $D_{2} \subseteq g\left(D_{0}\right)$. The proof is complete.

\section{ACKNOWLEDGEMENT}

The authors express sincere gratitude to both referees for a thoughtful reading of the paper and useful suggestions that helped to improve the presentation.

\section{REFERENCES}

[1] S. Banach and S. Mazur, Über mehrdeutige stetige Abbildungen, Studia Math. 5 (1934), 174-178

[2] F.E. Browder, Covering spaces, fiber spaces and local homeomorphisms, Duke Math. J. 21 (1954), 329-336 MR0062431 (15:978a)

[3] J. Cronin, Fixed Points and Topological Degree in Nonlinear Analysis, Mathematical Surveys and Monographs, vol. 11, American Mathematical Society, Providence, RI, 1964 MR0164101 $(29: 1400)$

[4] J. Dugundji and A. Granas, Fixed Point Theory I, Monogr. Matem., 61, PWN, Warszawa, $1982 \operatorname{MR} 0660439$ (83j:54038) 
[5] A. Gasull, J. Llibre, and J. Sotomayor, Global asymptotic stability of differential equations in the plane, J. Differential Equations 91 (1991), 327-335 MR1111178 (92j:34102)

[6] W.B. Gordon, On the diffeomorphisms of Euclidean space, Amer. Math. Monthly 79 (1972), 755-759 MR0305418(46:4548)

[7] J. Hadamard, Sur les transformations ponctuelles, Bull. Soc. Math. France 34 (1906), 71-84 MR 1504541

[8] J.K. Hale, Ordinary Differential Equations, Wiley-Interscience, New York, 1969 MR0419901 $(54: 7918)$

[9] P. Hartman, Ordinary Differential Equations, Wiley \& Sons, New York-London-Sydney, 1964 MR0171038 (30:1270)

[10] F. John, On quasi-isometric mappings I, Comm. Pure Appl. Math. 21 (1968), 77-110 MR0222666 (36:5716)

[11] J.W. Lee and D. O'Regan, Topological transversality. Applications to initial value problems, Ann. Pol. Math. 48 (1988), 247-252 MR0978675 (90c:34003)

[12] P. Lévy, Sur les fonctions des lignes implicités, Bull. Soc. Math. France 48 (1920), 13-27 MR 1504790

[13] H.X. Li, J. Liang, and T.J. Xiao, Criteria for the global invertibility of $C^{1}$ functions between Banach spaces, Nonlin. Anal. 51 (2002), 189-195 MR1918339 (2003f:47111)

[14] G. De Marco, G. Gorni, and G. Zampieri, Global inversion of functions: an introduction, NoDEA Nonlinear Differential Equations Appl. 1 (1994), 229-248 MR.1289855(95h:58014)

[15] L. Markus and H. Yamabe, Global stability criteria for differential systems, Osaka Math. J. 12 (1960), 305-317 MR0126019 (23:A3316)

[16] R. Nevanlinna, Über die Methode der sukzessiven Approximationen, Ann. Acad. Sci. Fenn. Ser. A.I. No. 291 (1960), 10 pp. MR0120534(22:11286)

[17] C. Olech, On the global stability of an autonomous system on the plane, Contrib. Differential Eq. 1 (1963), 389-400 MR0147734 (26:5248)

[18] C. Olech, On the Ważewski equation, Proceedings of the Conference "Topological Methods in Differential Equations and Dynamical Systems" (Kraków-Przegorzały, 1996), Univ. Iagel. Acta Math. 36 (1998), 55-64 MR.1661322 (99k:34002)

[19] G. Peano, Démonstration de l'intégrabilité des équations différentielles ordinaires, Math. Ann. 37 (1890), 182-228 MR.1510645

[20] R. Plastock, Homeomorphisms between Banach spaces, Trans. Amer. Math. Soc. 200 (1974), 169-183 MR0356122(50:8593)

[21] B. Porciau, Global invertibility of nonsmooth mappings, J. Math. Anal. Appl. 131 (1988), 170-179 MR0934439 (89f:58014)

[22] W. Rudin, Principles of Mathematical Analysis, third ed., McGraw-Hill, Inc., New York, 1976 MR0385023 (52:5893)

[23] J. Sotomayor, Inversion of smooth mappings, Z. Angew. Math. Phys. 41 (1990), 306-310 MR:1045818 (91g:58021)

[24] J. Sotomayor, Stability properties of the inverse mapping domain, in Real and Complex Singularities (São Carlos, 1994), Pitman Res. Notes Math. vol. 333, Longman, Harlow, 1995 MR 1478508 (98j:58015)

[25] T. Ważewski, Sur l'evaluation du domaine d'existence des fonctions implicites réelles ou complexes, Ann. Soc. Pol. Math. 20 (1947), 81-125 MR0026100(10:106h)

[26] A. Wintner, On the exact limit of the bound for the regularity of solutions of ordinary differential equations, Amer. J. Math. 57 (1935), 539-540 MR.1507092

[27] G. Zampieri, Finding domains of invertibility for smooth functions by means of attraction basins, J. Differential Equations 104 (1993), 11-19 MR1224119(94e:26022)

[28] G. Zampieri and G. Gorni, On the Jacobian conjecture for global asymptotic stability, J. Dynamics Differential Equations 4 (1992), 43-55 MR.1150396 (93a:34063)

Department of Mathematics, University of Craiova, Al. I. Cuza 13, Craiova, Romania E-mail address: octaviangenghiz@yahoo.com

Department of Mathematics, Eastern Mediterranean University, Famagusta, TrnC, Mersin 10, Turkey

E-mail address: yuri.rogovchenko@emu.edu.tr 